\title{
MULTIMODAL AD RECALL PREDICTION BASED ON VIEWER'S AND AD FEATURES
}

\author{
A PREPRINT
}

\author{
Mariya Malygina \\ Department of Psychology \\ Higher School of Economics \\ Neurodata Lab, LLC \\ Moscow, Russia \\ mmalygina@hse.ru \\ Abduragim Shtanchaev \\ Neurodata Lab, LLC \\ Moscow, Russia \\ a.shtanchaev@neurodatalab.com
}

\author{
Marina Churikova \\ Neurodata Lab, LLC \\ Department of Higher Nervous Activity \\ Lomonosov Moscow State University \\ Moscow, Russia \\ m. churikova@neurodatalab.com
}

\author{
Olga Perepelkina \\ Neurodata Lab, LLC \\ Moscow, Russia \\ o.perepelkina@neurodatalab.com
}

August 19, 2020

\begin{abstract}
Ad recall is a commonly used measure of advertising effectiveness. Automatic prediction of advertising effectiveness will help to improve video advertising and optimize the process of ad creation. We present a novel multimodal approach to ad recall prediction for video-advertising based on viewer's features and ad features. In our experiment twenty people watched ads ( $\mathrm{n}=100$ in total). Ads have ground truth ad recall that was previously obtained in a field study. While people were watching ads, we recorded them with video camera, collected contact photolpletysmography and eye-tracking data, and also asked them to complete questionnaires. From these data we extracted "viewer's features" emotional, physiological and behavioral parameters. As well, we had "ad features" - target rating Point (TRP) and weighted target rating point (WTRP) metrics. To predict ad recall from these features a range of regression models were tested. Random Gaussian projection with Support Vector Machine showed the best performance (MAE=0.09, R=0.6).
\end{abstract}

Keywords Affective Computing · Computational Advertisement

\section{Introduction}

Predictions of advertising effectiveness provide insights that can be of value for specialists who create marketing strategies, yet such predictions are still difficult to measure. Ad effectiveness is estimated via various metrics (product recall, ad like, purchase intent, and others) [1]. Ad recall is an important one as it impacts brand and product awareness. We aim to investigate whether value of metrics of ad effectiveness, namely ad recall score, can be predicted automatically by video. In the present study we use both ad data and viewers' responses to predict ad recall score for TV ads of various mobile carriers.
There have been developed two major approaches for automatic prediction of ad effectiveness. The first approach is to construct a model based on features of a video itself: e.g. ad content [2], media reach/buying data [3], and on direct response advertising metrics such as click-through-rate and cost per click [4]. Further we will use the term "ad features" to refer to the data of this type. The second approach is to address viewers' impressions or behavioral patterns (i.e., gaze data gathered in shelf recall experiments based on a forced-choice paradigm [5]) and make predictions based on customer responses. Further we will use the term "subject features" to refer to viewers' responses. Tools for physiological, behavioral and facial expressions measurements draw attention of marketing and ad research professionals [6] and are 
commonly referred to neurophysiological methods. Neurophysiological methods are promising as they provide insights in nonverbal responses, while a large part of marketing research is based on self-reports. Nonverbal and physiological responses are more informative than selfreports in terms of time resolution and are less affected by social desirability. Physiological responses captured by eye tracking and heart rate monitoring tools and electroencephalography method were proven to be associated with higher-level constructs widely used in advertising research - such as attention, affect, or memory [7].

We assume that ads with high recall affect subjects differently than ads with low recall. In this paper we propose to apply subject features of physiological, behavioural and emotional responses toward the ad, along with selfreports, to predict ad recall score. We hypothesize that an algorithm that takes subject features into account would predict ad recall score with a lower mean absolute error (MAE) and higher correlation as compared with an algorithm that works with ad features only. Our assumptions include the following: 1) Model based on ad features allows to predict ad recall score; 2) Model based on subject features allows to predict ad recall score; 3 ) The proposed approach that combines ad features and subject features can achieve lower MAE and higher correlation in ad recall prediction when compared with the prediction that uses only ad features or subject features.

\section{Related work}

\section{Behavioral and physiological responses in advertising research.}

Behavioral and physiological responses to the content provide hints to predict consumer behavior [8]. Since emotional responses affect consumer choice [1], advertising creatives have been transformed into emotionally appealing videos rather than videos with rational messages [9]. A large part of current ad content is supposed to evoke certain emotional response to a message, thus it became frequently investigated. Recent studies suggest that emotionally engaging ads are associated with higher purchase intent [10], as well as high-arousal and positive content has a better chance to become more viral, if consider social networks [11]. The findings of metaanalysis on the impact of media context [12] indicate that ad viewers would more likely recall ads when they are placed in emotionally congruent media contexts, and when program liking is higher.

Rating prediction of ad videos. Over the past years, the accuracy of automatic recognition has been improved by combining features from multiple modalities in social signal processing domain [13].

In marketing research, gaze parameters are often investigated along with traditional methods of self-reports and interviews. Consumer neuroscience and neuromarketing research is known for using electroencephalography
(EEG) and brain scanning techniques such as functional magnetic resonance imaging (fMRT), along with neurophysiological methods like heart rate, galvanic skin response (GSR), and electromyography. Studies show that adding these and other nonverbal and physiological parameters to the model can improve the quality of prediction.

A combination of contact photoplethysmography (cPPG) and remote emotion recognition was applied to predict ad viewers' self-reported attention, engagement, and sentiment when watching video ads on smartphones [14]. Multimodal approach showed the average accuracy of $61.5 \%$ for binary classification among self-reported metrics. To predict self-reported valence scores, H. Gauba et al. [15] fused EEG response and global sentiment-rating of video ads extracted from comments that were available on the internet. The best root mean square error of 0.714 value was achieved with random forest regression tree. A number of views for Super Bowl commercials on YouTube was predicted by EEG response, heart rate variability, eye tracking and self-reported liking [6]. The multi-layer perceptron network showed $82.9 \%$ of correct predictions based on a four-level ranking: ads with less than one million visits, $1 \mathrm{M}-5 \mathrm{M}, 5 \mathrm{M}-10 \mathrm{M}$, and ads with more than ten million visits. V. Venkatraman et al. [7] compared traditional and neurophysiological methods (eye tracking, implicit measures, heart rate monitoring, GSR, EEG, fMRI), as they correspond to the metrics of ad effectiveness such as attention, affect, memory, and desirability, and gross ratings points obtained in-market. Results showed that neurophysiological measures improved predictions of advertising effectiveness, particularly fMRI explained the most variance in predicting market-level elasticities of the participants.

Considering multimodal prediction of ad recall, X. Zhang study [16] aimed to predict high and low ad recall scores with ad element-related eye movement behavior. Logistic regression has shown that product-related gaze duration and brand-related amount of fixations are associated with recall. In contrast, L. Suurkivi study of short YouTube ad videos [17] has not shown associations between facial emotions, gaze parameters and ad recall, while elements extracted from ad content - visual brand and product exposure - have shown associations with ad likability. In Super Bowl online ads research [6] eye tracking, HRV and EEG parameters made it possible to distinguish between recalled versus non-recall online ads. Ad recall was obtained 2 hours after viewing the ads. Thus, ads with high recall affect viewers differently than ads with low recall.

\section{Materials and Methods}

A mixed design was used. Each test subject watched and estimated 10 ads with different ad recall scores. Responses to the ads with different ad recall represented within-subject factor. Within-subjects design is common 
in neurophysiological research as physiological responses can be reliable within subjects but not necessarily between subjects. In addition to this, subjects were exposed to different sets of ads. 10 sets of ads in total formed a between-subject factor.

Participants. Subjects were healthy adults ( $\mathrm{N}=20$, $\mathbf{M}_{\text {age }}=21.55$, sd=2.92, 12 females) who met target audience requirements, as defined by the marketing company. Subjects were Russian-speaking and had middle and high income. Subjects received monetary compensations for participating in the study.

Stimuli. Subjects watched television commercials of mobile carriers, courtesy of the partner marketing company. We used 100 video ads in the study $\left(M_{\text {duration }}=28.4 \mathrm{sec}, \mathrm{sd}=7 \mathrm{sec}\right)$. Ads represented different digital products of six mobile companies, for example satellite television or internet services, or mobile apps. Advertising region was Russia, ads were produced in Russian language for Russian customers. Additionally, two videos unrelated to advertising were set as baselines. Videos were validated to elicit moderately positive valence and low arousal [18].

\subsection{Ad recall as a target}

Our target metric for prediction is ad recall. Ad recall metric generally represents how many people out of a hundred remember a particular ad. All ads set as stimuli were examined through field marketing survey prior to this study, so their ad recall scores were already known. Marketing survey covered 300 people per ad. People were asked whether they were familiar with a particular ad after 7-14 days this ad had been taken off-air. The ads were broadcasted on TV prior to the study during years 2016-2019. The results of the marketing research served as ground truth.

We sorted 100 ads by ad recall scores and divided them into 10 groups where group 1 included $10 \%$ of ads with the highest ad recall score, and group 10 consisted of 10 $\%$ ads with the lowest ad recall score. Then we randomly picked one ad from every percentile group to form a set of ads so that our subjects would watch ads with high, medium and low ad recall scores. Thus, each subject viewed 10 ads with different ad recall scores from the general pool.

\subsection{Procedure}

Study was held in laboratory conditions. At first, subjects signed inform consent and filled in a demographic questionnaire, then they underwent the procedures of attaching contact photoplethysmography (cPPG) sensor and eye tracker calibration. Subjects were instructed to watch attentively 12 videos in total: 1) an unrelated movie segment to help them focus on the experiment and record their baseline heart rate; 2) 5 video ads in random order;
Table 1: Resulting table. We narrow it down to four basis for classifying the features

\begin{tabular}{|c|c|c|}
\hline & Source of Features & Features sets \\
\hline $\begin{array}{r}\text { Ad } \\
\text { Features }\end{array}$ & Media placement & TRP, WTRP \\
\hline \multirow{3}{*}{$\begin{array}{r}\text { Subject } \\
\text { Features }\end{array}$} & Questionnaires & $\begin{array}{l}\text { Valence, arousal, ad like, ad } \\
\text { interest, expected ad recall, } \\
\text { sex, age, income. }\end{array}$ \\
\hline & Contact Sensors & $\begin{array}{l}\text { Fixations, saccades, } \\
\text { heart rate, SDHR, SDNN, } \\
\text { RMSSD, PNN50, HF, LF, } \\
\text { VLF. }\end{array}$ \\
\hline & Remote Sensing & $\begin{array}{l}\text { Emotions, blink rate, } \\
\text { reaction time, heart rate. }\end{array}$ \\
\hline
\end{tabular}

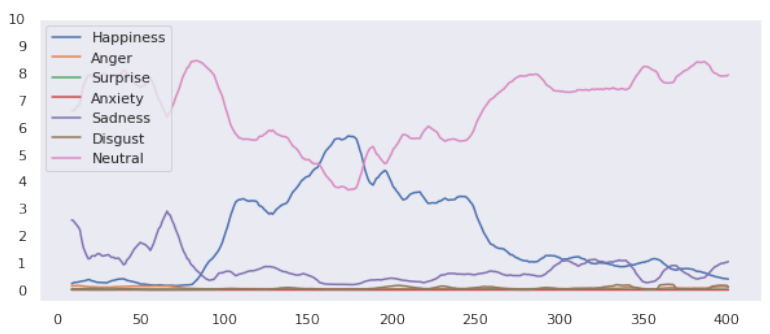

Figure 1: Example of the feature: automatically recognized emotions for one subject while she was viewing ad with high recall. Time axis is set in frames, one frame is $40 \mathrm{~ms}$.

3) an unrelated video with a relaxing sea view to let them take a break and record their baseline heart rate; 4) 5 remaining video ads in random order. Along with that, subjects were instructed to give verbal answers to the questions that were displayed on the screen after each ad. Verbal answers required a training stage: after the first baseline video subjects read the descriptions of the scales and gave answers to test questions orally. After answering the questions about the ad, subjects spent 15 seconds watching a mask - a neutral pic. Masks were relevant to telecommunication topic and helped to both support the context and let a respondent mask the impression from previous ad. Then they were proceeded to the next ad. 10 masks in total were used.

\subsection{Data recording}

Eye tracker and cPPG signal were recorded synchronously with video for each subject. We captured subjects during both ad watching and while giving verbal answers.

The videos were recorded with a webcam Logitech C920 in uncompressed bitmap format with a 1280x720 pixel resolution, and a $25 \mathrm{fps}$ frame rate. Subjects were lit by fluorescent ceiling lamps and sat in front of the cameras at a distance of about $70 \mathrm{~cm}$. 
Table 2: Summary of experiment results for Gaussian random projection with SVM model

\begin{tabular}{cccc|cc}
\hline \multicolumn{3}{c}{ Feature Sets } & \multicolumn{1}{c}{ MAE } & Pearson R \\
\hline- & Ad features & Remote Sensing & - & 0.0869 & 0.5901 \\
- & Ad features & - & - & 0.0866 & 0.5818 \\
Questionnaire & Ad features & - & Contact Sensors & 0.0865 & 0.5683 \\
Questionnaire & Ad features & - & - & 0.0874 & 0.5554 \\
Questionnaire & Ad features & Remote Sensing & Contact Sensors & 0.0894 & 0.5493 \\
Questionnaire & Ad features & Remote Sensing & - & 0.0889 & 0.5439 \\
Questionnaire & - & Remote Sensing & Contact Sensors & 0.1094 & 0.1865 \\
Questionnaire & - & Remote Sensing & - & 0.1094 & 0.1738 \\
- & - & Remote Sensing & Contact Sensors & 0.1083 & 0.1636 \\
- & - & Remote Sensing & - & 0.1113 & 0.1154 \\
\hline
\end{tabular}

Heart rate and Heart rate variability data was obtained with an optical pulse sensor Shimmer3 GSR+ attached to the subject's finger with sampling rate $256 \mathrm{~Hz}$.

Eye tracker GazePoint GP3 collected gaze data at $60 \mathrm{~Hz}$ with an 11-point calibration.

Questionnaire. For closed questions, researcher recorded each subject's verbal replies.

Ad features. Target rating point (TRP) and weighted target rating point (WTRP) metrics were provided as media placement reports. TRP represents the amount of target audience reached by TV [19] and is calculated as a sum of percents of people reached by a specific television program. TRP varied from $18 \%$ to $4081 \%$, mean $=1241 \%$. WTRP is a TRP weighted on the ad duration. WTRP shows what the reached target audience would be if the ad was 30 seconds long. WTRP was distributed from $9 \%$ to $3401 \%$ with mean $923 \%$.

Subject features. Subject features were obtained from the following steps of the study: 1) responses captured on video while subjects were watching ads; 2 ) responses captured on video when subjects were giving verbal answers; and 3) self-reports such as the questionnaire.

Automatic emotion recognition by video was performed with Neurodata Lab Emotion Recognition API 1 . with frame rate 25 . Anonymized API classified emotions by analyzing the holistic image of a face based on end-toend deep learning approach. Acquired emotion classes were: anger, anxiety, disgust, happiness, neutral, sadness, surprise as shown in Figure 11, the emotions' weights added up to 1 in each frame. We calculated mean weights of each emotion per recorded videos. Literature suggests that positive emotions are related to ad liking and higher brand recall [13].

Heart rate parameters. Parameters extracted from the time domain were: mean heart rate; standard deviation of heart rate (SDHR). These physiological parameters can be extracted both with cPPG and Neurodata Lab HeartTrack algorithm [20]. Also, we measured standard heart rate

\footnotetext{
${ }^{1}$ https://api.neurodatalab.dev/
}

variability components from cPPG - the square root of the sum of successive differences between adjacent RR intervals (RMSSD); percentage of successive RR intervals that differ by more than $50 \mathrm{~ms}$ (PNN50); standard deviation of normal-to-normal intervals (SDNN); and heart rate oscillations into very-low-frequency (VLF), low-frequency (LF), and high-frequency (HF) bands. Heart rate variability is applied to estimate valence, arousal and attention of ad viewers [13].

We calculated the following gaze parameters: the amount of fixations and the amount of saccades normalised on 30 seconds; amount of blinks in one minute. Longer fixations and smaller number of blinks are generally associated with higher visual attention and better memorising [21]. Besides, gaze ratio is correlated with preferences in advertisements [7].

The behavioral metric of reaction time was obtained with audio modality. Reaction times for the question "Did you like this ad? Give a yes or no answer" were calculated based on time between the question and the moment when the first "Yes" or "No" word appeared. Studies suggest that subjects answer faster if the stimuli corresponds to their actual impression [22].

Features obtained with self-report. Subjects evaluated each ad with ordinal scales varying from 1 (low) to 5 (high) scores depending on whether they liked the ad (ad like), whether it was boring or engaging (ad interest), and whether it was easy to remember (expected ad recall). Subjects estimated their own emotional state while watching the ad using scales of valence (positive or negative) and arousal (low or high) which varied from 1 to 9 [23]. We applied social-demographic data on sex, age and income of the subjects as well.

We grouped features in feature sets based on the type of technology that was used to obtain them. There groups are: questionnaire, contact sensors, remote sensing, and media report that provided us with ad features (as specified in Table 1). Specifically, our interest was to assess the contribution that contact and remote technologies gave in predictions. Using remote technologies only would allow us to shift to online marketing research. 


\section{Results}

\subsection{Data preprocessing}

Ad recall scores were unbalanced, scores distributed from $\min =0.15$ to $\max =0.86$, mean $=0.61$ with violating the assumption of normality (Shapiro-Wilk's W=0.95, p<.001).

All self-reported features were normalized with unitybased normalization (min-max feature scaling). Categorical features were converted to numerical using one hot encoding and labels encoder methods depending on a feature's structure. Other features which are described in the Table 1 were readily converted to numerical format by corresponding extraction algorithms. The omissions in the data were filled using second-order spline method column-wise.

\subsection{Algorithms}

Metrics. Metrics define the closeness of a predicted result to absolute truth that one sets forth. Thus, determining the right metrics plays a critical role in optimization task. In this work, mean absolute error (MAE) has been employed over root mean square error (RMSE) since its interpretation is more explicit, and variance associated with the frequency distribution of error magnitudes is not of interest. Mathematically MAE is defined as:

$$
M A E=\frac{1}{n} \sum_{j=1}^{n}\left|y_{j}-\hat{y}_{j}\right|
$$

Along with MAE, the Pearson correlation coefficient (PCC) metric was used to measure the correlation between predictions and the ground truth. The desired result of predictions should linearly correlate to the ground truth. Following is mathematical expression of PCC

$$
P C C=\frac{\sum_{j=1}^{n}\left(y_{j}-\bar{y}\right)\left(\hat{y}_{j}-\overline{\hat{y}}\right)}{\sqrt{\sum_{j=1}^{n}\left(y_{j}-\bar{y}\right)^{2}} \sqrt{\sum_{j=1}^{n}\left(\hat{y}_{j}-\overline{\hat{y}}\right)^{2}}}
$$

Models. In this work, a range of regression models have been analyzed, and the highest-scoring models were picked as final estimators. The original full features space yielded poor results. The reason for models' poor results, trained on the original feature space, might be conditioned by insufficiency of the data objects compared to the number of features. In that regard, a variety of random projection methods have been analyzed for this task. Empirically, Gaussian random projection [24] has shown to preserve the geometry of the original manifold, and therefore Gaussian random projection was used as a dimensional reduction method in the experiment. It should be noted that while availability of training objects increases, the need for dimensional reduction might depreciate. Additionally, Table 3 illustrates performance of each model. Time column stands for model training and
Table 3: Performance of all models

\begin{tabular}{lccc} 
Model & MAE & Pearson R & \multicolumn{2}{c}{ Time } \\
\hline GRP + SVM & 0.0894 & 0.5493 & $96.8 \mathrm{~ms} 8.69 \mathrm{~ms}$ \\
\hline LaSso & 0.0937 & 0.4882 & $70.7 \mathrm{~ms} 12.2 \mathrm{~ms}$ \\
\hline GRP + RF & 0.0981 & 0.4784 & $33.2 \mathrm{~s} 2.88 \mathrm{~s}$ \\
\hline XGBoost & 0.1011 & 0.4422 & $1.27 \mathrm{~s} 36 \mathrm{~ms}$ \\
\hline Stacking & 0.1005 & 0.4411 & $2.39 \mathrm{~s} 42.3 \mathrm{~ms}$ \\
\hline
\end{tabular}

inference time of each model. It it clear that Gaussian random projection with SVM provide highest scores in the shortest time. As a result, a combination of random Gaussian projection with eight components as a dimensionality reduction method and Support Vector Machine with RBF kernel as a classifier were used in the experiments. The data set contained 375 objects in total; therefore, as a means of effective data utilization, the leave-oneout-Group cross-validation method [25] was used to split test-train sets for evaluation.

\subsection{Experiments}

Model with all features. In this study, two kinds of experiments were conducted. Four groups of features were available, which are: Questionnaire self-report, Ad features, Remote Sensing, and Contact Sensors. More detailed descriptions are provided in "Data Recording" section, in Table 1. Firstly, the model was tested using all available features, including features obtained using contact sensors. The obtained results are presented in Table 2. As we can see from the table, it is surprising that inclusion of all features in model training did not result in the highest correlation. Table 3 illustrates how each model considered in this study performed on full feature set.

Models with different feature sets. In the second experiment, different subsets of features were employed. The experiment underlines the importance of each feature set in the model's predictions and identifies a possible shift to usage of Remote Sensing algorithms alone for ad recall score prediction. That means that ad recall score could be predicted only using the respondent's facial video that is being recorded while he/she is watching an add. The obtained results are summarised in Table 2 Figure 2 illustrates correlation between the ground truth ad recall score and the ad recall score predictions of our best model. The red line illustrates the highest possible correlation.

\section{Discussion}

From Table 2, a set of interesting observations can be made. The feature sets of interest are colored in blueish according to their Pearson Correlation Coefficient. The model trained with the full features set, contrary to the expectations, has not produced the highest coefficient $(\mathrm{R}=0.55)$. The highest Pearson correlation $(\mathrm{R}=0.59)$ resulted from a combination of ad features and remote 


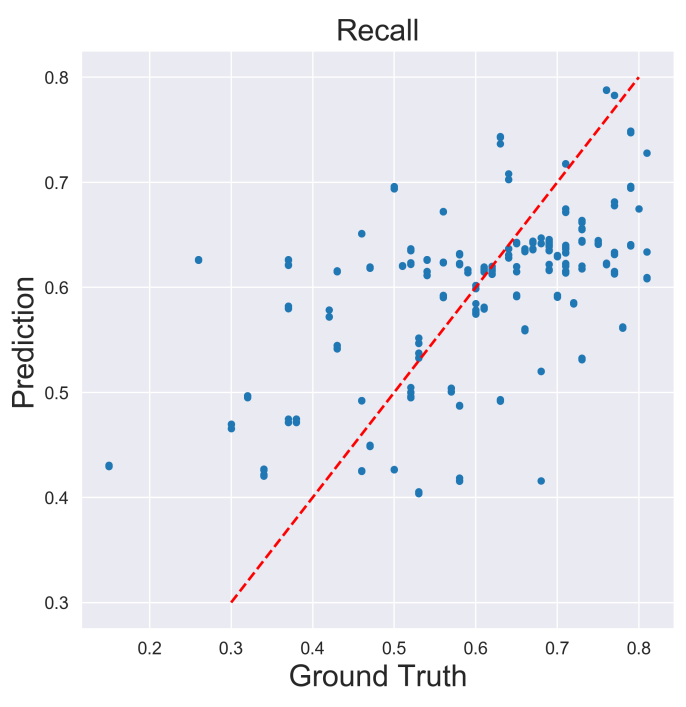

Figure 2: Correlation of the Best Estimation with the Ground Truth

sensing (emotional and physiological data extracted automatically from video feed). The next most significant result was yielded from analyzing the ad feature set alone. That means that the feature set (remote sensing) we generated has a considerable weight in the model's predictions. The combination of the ad features, questionnaire, and contact sensors has showed the lowest MAE (MAE=0.0865). We have found that ad features alone TRP and WTRP - can be used to predict ad recall scores $(\mathrm{R}=0.58, \mathrm{MAE}=0.0866)$. At the same time, subject features alone are not enough to make ad recall score predictions accurately, irrespective of whether these features are presented by self-report or physiological responses $(\mathrm{R}=0.19$, MAE=0.1094). MAE of ad recall and it's median value (0.63) equals to 0.1092 , which means that the predictions are as accurate as median values if ad features are not used.

Limitations and research directions. The main limitation is that ad recall scores were obtained from the different sample. Despite the general similarity of the two samples in terms of sex, age and income parameters, physiological responses vary considerably among individuals and cannot be fully extrapolated to other samples. That means that we could obtain different physiological data if marketing survey respondents would have participated in the present study. Along with that, the features we used in the proposed model may not be exhaustive. Thus, ad content, e.g. the presence of celebrities or known songs needs more detailed exploration in future. Since ad recall mainly refers to cognitive aspects of consumer behavior like increasing awareness about the brand, separate features associated with cognitive load processes should be included, for instance visual attention.

While the present research focuses on ad recall, it would be desirable to examine metrics like purchase intent di- rectly. We plan to make a step up from laboratory environment and get a more representative sample by collecting viewers' responses online.

\section{Conclusion}

In this paper we have presented the multimodal approach to predict ad recall score of video advertisements run on TV. The suggested approach combines ad features and subject features. We took a set of ads with ad recall scores measured within the framework of a field study after these ads had been taken off-air. In the present study, subjects watched these ads and answered a number of questions about each ad while we recorded them on video and registered their gaze and heart rate parameters. We have compared the following regression models: the one predicting ad recall based on ad placement data alone, and the other combining ad placement data with physiological and emotional parameters obtained from ad viewers.

The results are promising as the suggested approach has achieved lower MAE in ad recall scores prediction as compared with the prediction based on ad placement data only. Random Gaussian projection with Support Vector Machine was chosen as final estimators.

Ad recall score predicted for a particular ad allows to assess advertising prior to running it. Predictions made in the present study reflect how the ad would be actually remembered by the target audience (compared to ad recall obtained in classical ad pre-broadcasting focus groups), since ad recall scores that we used as ground truth were measured during the field study after the ads had been shown on TV. However, our results suggest that ad recall is strongly connected to media placement. Regression model that took into account subject features alone have shown considerably lower correlation with ground truth and higher MAE. Thus, using the proposed approach for ad recall prediction, one could estimate media placement volume required to achieve desired ad recall score.

Remote sensing features, namely, emotions, heart rate, blink rate and reaction time extracted with neural networks have shown the best performance among other subject features. A combination of ad features and subject features obtained with remote sensing has given the highest Pearson correlation. This result identifies a possible consideration of a shift to a usage of remote sensing algorithms in marketing and advertising research.

A major benefit of self-report in marketing studies is that it enables access to a very large representative sample [26] and remains cost efficient. The use of remote technologies that detect emotional and physiological parameters overcome the general limit of neuromarketing research, namely small numbers of participants examined in a laboratory conditions [27]. Remote sensing requires a webcam only, thus it allows to collect data from a large number of respondents in short term, and enhance performance of predictions at the same time. 
These results open up new horizons as they suggest that the proposed multimodal approach helps to make the predictions of ad recall more accurate.

\section{References}

[1] Martin Eisend and Farid Tarrahi. The effectiveness of advertising: A meta-meta-analysis of advertising inputs and outcomes. Journal of Advertising, 45(4):519-531, 2016.

[2] Yuheng Li, Yiping Zhang, and Ruixi Yuan. Measurement and analysis of a large scale commercial mobile internet tv system. In Proceedings of the 2011 ACM SIGCOMM conference on Internet measurement conference, pages 209-224, 2011.

[3] David W Cowling, Mary V Modayil, and Colleen Stevens. Assessing the relationship between ad volume and awareness of a tobacco education media campaign. Tobacco Control, 19(Suppl 1):137-142, 2010.

[4] Ritu Lohtia, Naveen Donthu, and Edmund K Hershberger. The impact of content and design elements on banner advertising click-through rates. Journal of advertising Research, 43(4):410-418, 2003.

[5] Klaus Duerrschmid and Lukas Danner. Eye tracking in consumer research. In Methods in Consumer Research, Volume 2, pages 279-318. Elsevier, 2018.

[6] Jaime Guixeres, Enrique Bigné, Jose M Ausín Azofra, Mariano Alcañiz Raya, Adrián Colomer Granero, Félix Fuentes Hurtado, and Valery Naranjo Ornedo. Consumer neurosciencebased metrics predict recall, liking and viewing rates in online advertising. Frontiers in psychology, 8:1808-1019, 2017.

[7] Vinod Venkatraman, Angelika Dimoka, Paul A Pavlou, Khoi Vo, William Hampton, Bryan Bollinger, Hal E Hershfield, Masakazu Ishihara, and Russell S Winer. Predicting advertising success beyond traditional measures: New insights from neurophysiological methods and market response modeling. Journal of Marketing Research, 52(4):436-452, 2015.

[8] Neale Martin and Kyle Morich. Unconscious mental processes in consumer choice: Toward a new model of consumer behavior. Journal of Brand Management, 18(7):483-505, 2011.

[9] Joanna Cartwright, Helen McCormick, and Gary Warnaby. Consumers' emotional responses to the christmas tv advertising of four retail brands. Journal of Retailing and Consumer Services, 29:82-91, 2016.

[10] Jieun Lee and Ilyoo B Hong. Predicting positive user responses to social media advertising: The roles of emotional appeal, informativeness, and creativity. International Journal of Information Management, 36(3):360-373, 2016.
[11] Irina Heimbach and Oliver Hinz. The impact of content sentiment and emotionality on content virality. International Journal of Research in Marketing, 33(3):695-701, 2016.

[12] Eun Sook Kwon, Karen Whitehill King, Greg Nyilasy, and Leonard N Reid. Impact of media context on advertising memory: A meta-analysis of advertising effectiveness. Journal of Advertising Research, 59(1):99-128, 2019.

[13] Genki Okada, Kenta Masui, and Norimichi Tsumura. Advertisement effectiveness estimation based on crowdsourced multimodal affective responses. In Proceedings of the IEEE Conference on computer vision and pattern recognition workshops, pages 1263-1271, 2018.

[14] Phuong Pham and Jingtao Wang. Understanding emotional responses to mobile video advertisements via physiological signal sensing and facial expression analysis. In Proceedings of the 22nd International Conference on Intelligent User Interfaces, pages 67-78, 2017.

[15] Himaanshu Gauba, Pradeep Kumar, Partha Pratim Roy, Priyanka Singh, Debi Prosad Dogra, and Balasubramanian Raman. Prediction of advertisement preference by fusing eeg response and sentiment analysis. Neural Networks, 92:77-88, 2017.

[16] Xuebai Zhang and Shyan-Ming Yuan. An eye tracking analysis for video advertising: Relationship between advertisement elements and effectiveness. IEEE Access, 6:699-707, 2018.

[17] Liisi Suurkivi et al. Relationship between ad elements, recall and likeability in short Youtube ads. PhD thesis, Tartu Ülikool, 2019.

[18] Lisanne M Jenkins and David G Andrewes. A new set of standardised verbal and non-verbal contemporary film stimuli for the elicitation of emotions. Brain Impairment, 13(2):212-227, 2012.

[19] Paul W Farris, Neil Bendle, Phillip E Pfeifer, and David Reibstein. Marketing metrics: The definitive guide to measuring marketing performance. Pearson Education, 2010.

[20] Mikhail Artemyev, Marina Churikova, Mikhail Grinenko, and Olga Perepelkina. Robust algorithm for remote photoplethysmography in realistic conditions. Digital Signal Processing, pages 102-111, 2020.

[21] Taisuke Imai, Min Jeong Kang, and Colin F Camerer. When the eyes say buy: visual fixations during hypothetical consumer choice improve prediction of actual purchases. Journal of the Economic Science Association, 5(1):112-122, 2019.

[22] Hanne Stenzel, Philip JB Jackson, and Jon Francombe. Speech reaction time measurements for the evaluation of audio-visual spatial coherence. In 2017 Ninth International Conference on Quality of 
Multimedia Experience (QoMEX), pages 1-6. IEEE, 2017.

[23] Margaret M Bradley and Peter J Lang. Measuring emotion: the self-assessment manikin and the semantic differential. Journal of behavior therapy and experimental psychiatry, 25(1):49-59, 1994.

[24] Xiaoli Fern and Carla Brodley. Random projection for high dimensional data clustering: A cluster ensemble approach. Proc 20th Int'l Conf Machine Learning, pages 186-193, 012003.

[25] Tzu-Tsung Wong. Performance evaluation of classification algorithms by $\mathrm{k}$-fold and leave-one-out cross validation. Pattern Recognition, 48, 092015.

[26] Adam Hakim, Shira Klorfeld, Tal Sela, Doron Friedman, Maytal Shabat-Simon, and Dino J Levy. Pathways to consumers minds: Using machine learning and multiple eeg metrics to increase preference prediction above and beyond traditional measurements. bioRxiv, pages 1-34, 2018.

[27] Adam Hakim and Dino J Levy. A gateway to consumers' minds: Achievements, caveats, and prospects of electroencephalography-based prediction in neuromarketing. Wiley Interdisciplinary Reviews: Cognitive Science, 10(2):e1485, 2019. 